\title{
Clinical use of electronic portal imaging to analyse tumor motion variation during a 3D-conformal prostate cancer radiotherapy using online target verification and implanted markers
}

\author{
George Felix Acquah1, Magnus Gustavsson³, Chris Osam Doudoo', \\ Richard Kwabena Agbeve', Bernhard Schiestl ${ }^{1,2}$ \\ ${ }^{1}$ Department of Radiation Oncology, Sweden Ghana Medical Centre, Cantonments, Accra, Ghana. \\ ${ }^{2}$ Department of Therapeutic Radio Oncology, University Hospital of Innsbruck, Innsbruck, Austria. \\ ${ }^{3}$ Department of Medical Physics, Sahlgrenska University Hospital, Gothenburg, Sweden.
}

Received May 29, 2014; Revised July 24, 2014; Accepted July 26, 2014; Published Online September 01, 2014

\section{Original Article}

\begin{abstract}
Purpose: To evaluate the daily treatment setup variation and the interfraction and intrafraction prostate motion with portal imaging and implanted fiducial markers during irradiation with a 3D conformal radiotherapy for localized prostate cancer patients. Methods: By remote verification, shifts from isocenteric positioning and inter/intra-fraction prostate motion were investigated for 34 patients treated supine with escalated dose conformal radiotherapy. To limit the effect of inter-fraction prostate motion, patients were planned and treated with an empty rectum and a comfortably full bladder. Daily pre-therapy and treatment electronic portal images were obtained for anterior and lateral treatment fields according to an on-line target localization protocol using three gold markers. From these images, random and systematic set-up errors were measured by matching corresponding patients' gold markers on reference digitally reconstructed radiographs (DRR). Superior-inferior, anterior-posterior and lateral motions were measured from the displacement of the gold markers implanted into the prostate before planning. A planning target volume (PTV) was derived to account for the measured prostate motion and field placement deviations. Results: Analysis of 1,278 portal images to determine changes in the radiation field during the course of treatment. From the data, random isocenter positioning deviations were $2.66 \mathrm{~mm}, 2.78 \mathrm{~mm}$ and $2.59 \mathrm{~mm}$ for vertical, lateral and longitudinal movements respectively. The systematic deviations were $3.15 \mathrm{~mm}, 3.09 \mathrm{~mm}$ and $2.52 \mathrm{~mm}$ for vertical, lateral and longitudinal movements respectively. From the verification process, it was realized that $44.7 \%$, $42.8 \%$ and $31.4 \%$ of the vertical, lateral and longitudinal prostate migrations respectively needed correction/shift. Conclusion: Random set-up errors were small using real-time isocenter placement corrections. Inter-fraction prostate motion remained the largest source of treatment error, and observed motion was greatest at the laterals. In the absence of real-time pre-treatment imaging of the prostate position, using sequential portal films of implanted gold markers, portions of the PTV is missed and surrounding tissues not spared. This research improves quality assurance by confirming the prostate position within the treatment field over the course of therapy.
\end{abstract}

Keywords: Gold Markers; Prostate Motion; Systematic and Random Errors; Portal Imaging; EPID; DRR

\section{Introduction}

The encouraging results of external beam dose escalation trials for localized prostate cancer patients $1,2,3,4$ have prompted a re-examination of radiation treatment techniques to establish the best method of safely escalating the dose, without unduly compromising the treatment volume. Radia-

Corresponding author: George Felix Acquah; Department of Radiation Oncology, Sweden Ghana Medical Centre, Cantonments, Accra, Ghana.

Cite this article as:

Acquah GF, Gustavsson M, Doudoo CO, Agbeve RK, Schiestl B. Clinical use of electronic portal imaging to analyze tumor motion variation during a 3D-conformal prostate cancer radiotherapy using online target verification and implanted markers. Int $J$ Cancer Ther Oncol 2014; 2(4):02044. DOI: 10.14319/ijcto.0204.4

(C) Acquah et al. tion treatments always starts with the acquisition of planning images ${ }^{5}$ and its efficacy depends on the accuracy of patient setup at each daily fraction. The problem is to reproduce the patient position and prostate location at the time of acquiring the planning scans for each fraction of the treatment process. The PTV includes the Clinical Target Volume (CTV) and associated treatment uncertainties which includes but not limited to imaging, patient setup, and organ motion. ${ }^{6}$ To analyse the daily movements of the prostate, gold markers are implanted in the prostate and portal images taken and manually matched with reference images to locate the prostate. 
Geometrical and fiducial markers are annotated onto a highly quality generated DRRs, that are compared with electronic portal images (EPIs) acquired right before treatment dose delivery. Portal images are acquired using an electronic portal imaging device (EPID). ${ }^{5}$

The largest motion was observed to be in the anterior/posterior (AP) direction and to a lesser extent in the superior/inferior (SI) direction. The AP movement was strongly correlated with rectal filling and then to a lesser extent with bladder filling., 8 The management of target localization emanates in the concept of treatment margins, described in the ICRU reports 50 and 62.9, 10 The aim of imaging for treatment verification is to evaluate the geometric uncertainties both due to organ motion and setup variations through image guided radiotherapy (IGRT). ${ }^{11}$ Portal imaging using bony structures can only detect set-up variations, if implanted markers are used; both set-up variations and internal organ motion can be detected and if necessary, corrected. ${ }^{12}$ Two types of deviations have to be distinguished: the day-to-day (random) variations and the systematic variations, present during all treatment fractions. ${ }^{13}$ Image-guidance strategies used to reduce setup error are generally classified as either online or offline procedures. An online approach acquires and assesses information from daily imaging, typically before every treatment fraction. Simple corrections are implemented to compensate for noted deviations in patient's position that exceed a predefined threshold before radiation delivery. An offline strategy refers to frequent acquisition of images without immediate intervention. ${ }^{14}$ In general, online correction strategies achieve a larger reduction in geometric errors than offline approaches, but at the expense of more effort and time at treatment delivery and a higher imaging dose.

In prostate radiotherapy $7,15,16,17,18,19,20$, the actual position of the prostate can be visualized and verified by using gold markers as surrogates. The use of implanted markers and electronic portal imaging has become the gold standard for daily verification of position and correction of patient setup and organ displacement errors ${ }^{21,22,23}$ and is the focus of this article.

\section{Methods and Materials}

In this study, gold markers were implanted into prostate cancer patients $(\mathrm{N}=34)$ who received external radiation therapy with $3 \mathrm{D}$ conformal radiotherapy for treatment. To verify the treatment position of the prostate, portal images $(\mathrm{N}=1,278)$ representing the displacements were acquired with an iViewGT ${ }^{\mathrm{TM}}$ electronic portal imaging device. ${ }^{24}$ To analyse patient and prostate position variations, the marker contours derived from the DRR, were displayed overlaying the portal image and manually matched. The random error in the prostate position, derived from the gold markers, is defined as the Standard deviation (SD) of the day-to-day variations, averaged over all patients in the group. The systematic error is defined as the SD of the distribution of average prostate deviations per patient. For inter-fraction prostate position corrections for both systematic and random errors, an on-line correction procedure is applied. After manual alignment of the marker annotations onto the portal images, the set-up deviations and required corrections are displayed on iView matching software. Prostate motions in ventral-dorsal, cranial-caudal and left-right directions are corrected and intra-fraction prostate deviations checked again, before the irradiation dose is administered.

\section{Radiation Dose Planning Technique}

Each patient had three fine cylindrical gold markers $(1 \times 8$ $\mathrm{mm}$ ) inserted trans-rectally into the prostate (to serve as a surrogate for the prostate) under ultrasound guidance by an urologist before treatment planning. Patients were scanned supine, with their hips immobilized from the waist to mid-thigh in a rigid foam cradle and with the lower legs supported in a soft foam immobilization device. Planning CT images were taken at $3 \mathrm{~mm}$ slice thickness from the top of the first lumbar vertebrae to below the pelvis. Patients were asked to empty their bowels (taking 10mg of dulcolax) 2 days prior to scanning, and their bladder was kept comfortably full by drinking two glasses of water mixed with $18 \mathrm{mls}$ of gastrografin 30 min prior to the scan. Planning Magnetic resonance imaging (MRI) scans are also taken and fused with that of the CT scans. The prescribed dose to the isocentre was 78 Gy in 39 fractions over 7.8 weeks.

\section{Radiation Treatment Technique}

The first 4 and 2 monitor units (MUs) of the lateral and anterior treatment fields respectively were used to take portal images before each fraction; the gold markers from the DRRs were manually matched unto the images. A shift threshold of $3 \mathrm{~mm}$ was used for the prostate migration corrections and any translations of more than that were corrected before each treatment. The setup error is estimated and the patient position is corrected to reduce the discrepancies between the localization and reference images. The anterior treatment fields are used to locate the lateral and longitudinal prostate motion whiles the lateral treatment fields, are used for the vertical prostate motion. After the corrections are done through repositioning of the patients, a verification image is taken to confirm the shift before the actual treatment is given.

\section{The iView GT ${ }^{\mathrm{TM}}$ Electronic Portal Imaging Device}

A standard configuration of the iViewGT ${ }^{\mathrm{TM}}$ portal imaging device includes an amorphous silicon (a-Si) flat mega voltage (MV) panel image detection system with powerful image processing and display, using industry standard IT hardware and software. Under computer control, the fully automated image acquisition and comprehensive analysis functions are 
presented via a graphical user interface with a single monitor at the linear accelerator control area. It provides fast radiotherapy treatment verification for improved patient throughput. The image is taken when X-rays passing through the patient activate a fluorescent screen and the amorphous silicon panel captures the resultant light. The dose required is low; typically 2 to 4 MUs. iViewGT uses its own dose-monitoring system to ensure that the optimum dose from the fluorescent screen and amorphous silicon panel is used to acquire the portal image. Any displayed image may be zoomed up to maximum full screen width for closer examination. Images can also be displayed in the most appropriate form; window and level gray scale controls, edge enhancement for anatomical sites with less density differential, and lung and bone inversion for soft tissue identification. Images can be enlarged, scaled, measured and flipped for better comparison. The imager had sufficient resolution to reliably identify the fiducial markers.

\section{Analysis of portal imaging data}

Each patient contributed between a range of 34-37 portal images, and the quality of the images ware excellent and fiducial markers were clearly identified (Figure 1). The images were compared to the corresponding DRRs after appropriate demagnification, window settings and grey level scale were applied. Systematic errors in prostate displacement were calculated as the average of the means of the measured prostate displacements for each patient. After aligning of the marker positions onto the reference images as shown in Figure 1, the set-up deviations corrections are displayed and an on-line correction procedure applied. Lateral (+ sign for left shift and - sign for right shift), longitudinal (+ sign for superior and - sign for inferior shift) and vertical shifts (+ sign for anterior shift and - sign for posterior shift) are done manually to correct these random and systematic errors before the actual treatment doses are administered. Random errors were calculated as the average tendency for errors to occur about each mean displacement error. No markers were lost or marker migrations after verification of more than $3.0 \mathrm{~mm}$ (measured in two planes) were observed for any patient.

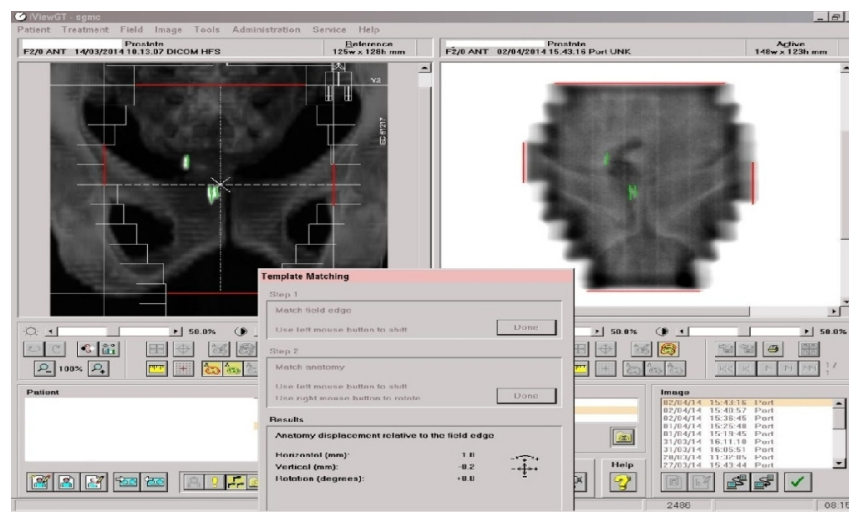

FIG. 1: Gold markers (green) and field edge (red) annotations on the DRR and EPI.

\section{Results}

The lateral and anterior treatment fields are used to calculate prostate shifts for all prostate cancer patients undergoing treatment at the Sweden Ghana Medical Centre (SGMC), using the iViewGT portal imaging device. From the analysis, the random error, defined as the standard deviation of the day-to-day variations, averaged over all patients in the group and the systematic error defined as the standard deviation of the distribution of average prostate deviations per patient were calculated. The results are presented in Table 1 together with their maximum deviations. To analyze the data, all negative signs were ignored to give the true or actual magnitude of deviation regardless of the direction of the gold markers. Our treatment technique did not permit measurement of rotational set-up errors. The mean representing the systematic deviations were observed to be associated with errors transferred from the Treatment Planning System (TPS) to the Linear accelerator (Linac) or errors in the iView system itself. The standard deviation, representing the random deviations denotes the gold marker (prostate) movements and errors in patient setup. The SD values are very close to the mean values, meaning that the gold markers were concentrated around the mean value.

TABLE 1: Components of isocentric positioning error in 34 prostate cancer patients.

\begin{tabular}{cccc}
\hline \hline Error type $(\mathrm{mm})$ & MAX & Systematic & Random \\
\hline VERT & 16.0 & 3.15 & 2.66 \\
LAT & 19.5 & 3.09 & 2.78 \\
LONG & 15.5 & 2.52 & 2.59 \\
\hline \hline
\end{tabular}
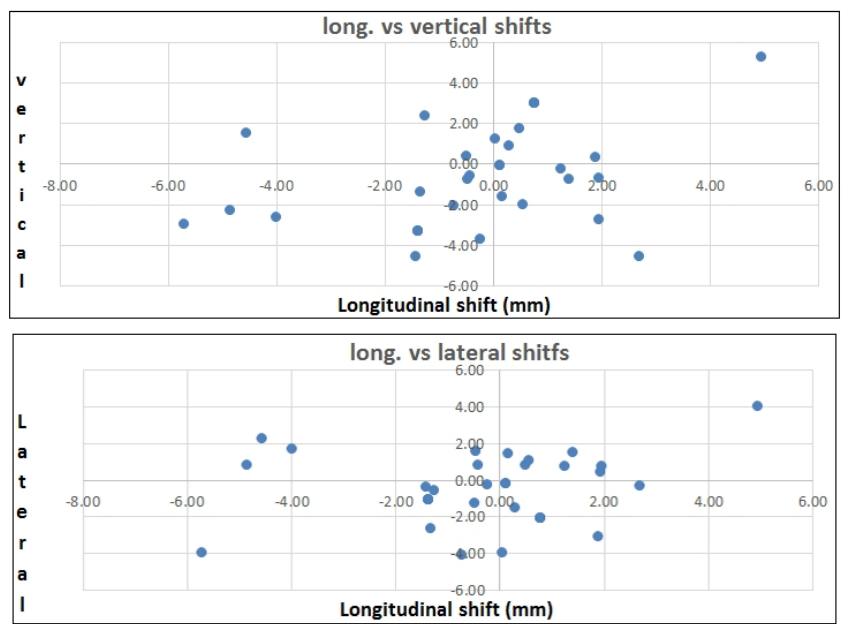

FIG. 2: Statistical analysis of gold marker movements in the prostate during daily setup.

The results (in $\mathrm{mm}$ ) are prostate position relative to isocenter systematic deviation and the shifts are isocenter shifts not couch shifts. 


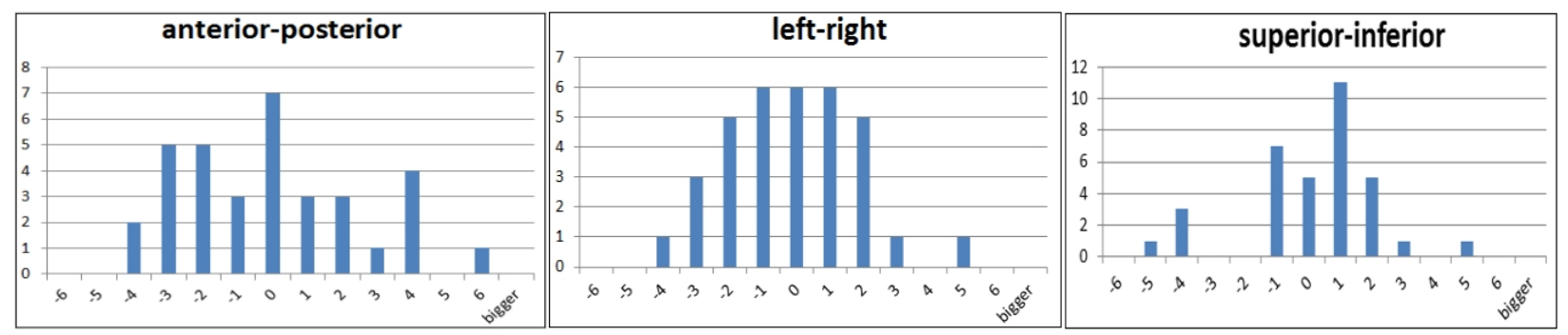

FIG. 3: Histograms showing the frequency distribution of prostate motion in the various planes.

Implanted fiducial markers allow detection and correction of organ and/or tumour specific position variations. This image guided radiotherapy procedure with gold markers and the EPID has resulted in accurate high-dose, high-precision prostate treatments, with less toxicity to the bladder and rectum. Depending on accuracy requirements, off-line correction protocols appear to be effective in reducing systematic deviations at an acceptable workload. With on-line corrections a very high geometric accuracy $(<3 \mathrm{~mm})$ can be obtained, but at the cost of a considerably larger effort. These results provided quantitative inter and intra-fractional analysis of the prostate motion and its individual gold markers.

\section{Discussion}

At SGMC, so far, three fine gold markers are implanted in prostate cancer patients who received external radiation therapy with 3D conformal radiotherapy planning technique for treatment. To verify the treatment position of the prostate using an iViewGT verification system, portal images are acquired with an electronic portal imaging device, using the first 6 monitor units (MUs) of the treatment beams.

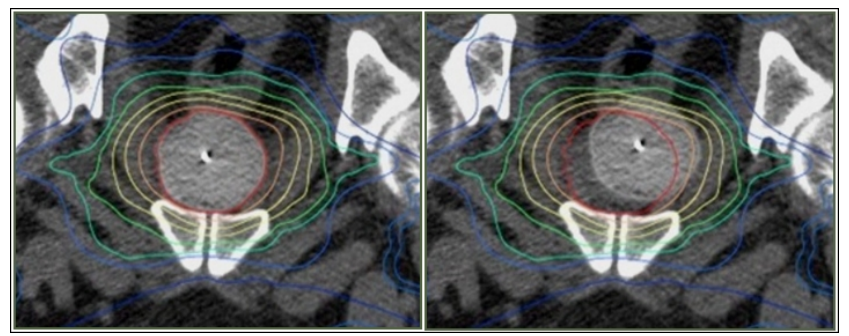

FIG. 4: Two planning images demonstrating an example of prostate movement during the treatment process with respect to the reference prostate position.

The gold marker errors identified in this study compare very favorably to our earlier experience with a bony anatomy matching technique for prostate cancer radiotherapy. Systematic errors have been reduced from an average of 4.34 to $2.92 \mathrm{~mm}$ and random errors from 3.95 to $2.68 \mathrm{~mm}$. This is likely attributable to the use of gold markers and the online corrections used to treat current prostate cancer patients. The results confirm that prostate motion is the major source of error in radiation treatment delivery for prostate cancer. Average prostate motion ranged from $4.54 \mathrm{~mm}$ posteriorly to
$5.30 \mathrm{~mm}$ anteriorly, and $5.72 \mathrm{~mm}$ inferiorly to $4.93 \mathrm{~mm} \mathrm{su}-$ periorly. These ranges are similar to those observed by these authors. ${ }^{11,16,18,24}$ However, despite the large extremes of motion encountered in some of our patients, the SD for prostate motion overall was still very narrow, and over $60 \%$ of observed movements were actually $3 \mathrm{~mm}$ or less. This implies $39.6 \%$ of observed patient positioning setup needs shifts or corrections prior to treatments. Verification images taken for all treatments fields after the setup correction was done were below $3 \mathrm{~mm}$. Without the use of this daily gold marker matching, means the patients would have been irradiated outside the target.

The results of current studies designed to limit set-up errors and extents of prostate motion are encouraging and demonstrate true advantages over matching on bony structures annotation techniques. Overall, the observed prostate motion tend towards the anterior direction, likely because an empty rectum protocol which ensures that the prostate was planned while lying in a posterior position. Prostate motion was greatest also at the base (inferior), and the least at the apex (superior). Set-up deviations in lateral, longitudinal and vertical directions are corrected and checked again, before the irradiation. These represent the intrafraction prostate motion during the course of the treatments. These shifts or corrections were less than the action level set in all possible directions for all our 34 patients used for this study.

This method assumes a high degree of correlation between the prostate target and reference target location in real time. This reduction in errors due to target motion location permits the use of high precise doses to the target, which in turn reduce dose to normal tissues. Ideally, the errors from patient setup and target motion should be simultaneously corrected for before treatment, with real-time portal image screening of implanted gold markers. The newest generation of amorphous silicon flat plate imaging device proved to be adequate for this purpose. The portal images provided excellent resolution of the gold markers, while real time corrections were done to identify the patients with extreme prostate motion, and permit corrective action to be taken daily during the course of treatment. 


\section{Conclusion}

A 3DCRT treatment technique for localized prostate cancer that uses fiducial markers, on-line isocentre verification, and patient instruction concerning bladder and rectal protocol resulted in small random and systematic set-up errors as well as inter/intra-fraction prostatic motion. The results confirmed that our dose escalation technique for treating prostate cancer patients are safe since the actual position of the target can be located on a daily basis.Higher radiation dose levels have been shown to be associated with improved tumor-control outcomes in localized prostate cancer patients. They are also consistently associated with improved biochemical control outcomes and reduction in distant metastases. Such reductions reduce the risk of radiation injury to the bladder base and the bulb of the penis, or permit additional dose to be given safely to the prostate. Our highly conformal technigue gives less risk of rectal, bladder and femoral heads toxicity because of the greater precision in treatment set-up and dose delivery.

The prostate, known to move independently of bony landmarks, requires additional effort to visualize its position prior to radiation delivery. Patient instruction intended to limit rectal and bladder volumes before treatment may have reduced the frequency of large prostate movements, but did not eliminate them. The use of gold markers and electronic portal imaging is the gold standard for daily verification of position and correction of patient setup and organ displacement errors. This report summarizes our experience with this technique and also compares our formal way of treating prostate cancer without markers but relaying on bony anatomy for matching. It was noted that, systematic errors reduced from an average of 4.34 to $2.92 \mathrm{~mm}$ and random errors from 3.95 to $2.68 \mathrm{~mm}$ with our current treatment technique. The tolerance for the bony anatomy technique was set to $5 \mathrm{~mm}$ to compensate for the movement of the prostate without the gold markers. Approximately $39.6 \%$ of observed patient positioning setup (using gold markers) needs corrections prior to treatments as compared $56.8 \%$ when matched on bony anatomy.

In conclusion, the need to use interventions (e.g. IGRT) depends on patient specific motion and treatment geometries. Surrogate position/movement information (e.g. implanted markers) play a key role in this area, both validating observations from 3D patient models, as well as potentially providing data of acceptable state for treatment.

\section{Conflict of interest}

The authors declare that they have no conflicts of interest. The authors alone are responsible for the content and writing of the paper.

\section{Acknowledgements}

This manuscript is based on work done at the Sweden Ghana Medical Centre, Accra, Ghana from March 2012 to April 2014. We wish to express our gratitude to our oncologists (especially Olof Stahl, Jens Engleson, Clement Edusa and Hannah Simonds), dosimetrists, radiotherapists (especially Eva Zuiderveld, Maud Bempeh, Promise Ahiagbenyo and Charne Martin), radiographers, and other members of the Oncology team of SGMC (especially Michael Odonkor) for their contributions towards this work. This work is also dedicated to the memory of the late Mr. Emmanuel Kwaku Nani (a pioneer of Medical Physics in Ghana).

\section{References}

1. Hanks GE, Hanlon AL, Schultheiss TE, et al. Dose escalation with $3 \mathrm{D}$ conformal treatment: five year outcomes, treatment optimization, and future directions. Int J Radiat Oncol Biol Phys 1998; 41:501-10.

2. Pollack A, Zagars GK. External beam radiotherapy dose response of prostate cancer. Int J Radiat Oncol Biol Phys 1997; 39:1011-8.

3. Zelefsky MJ, Leibel SA, Gaudin PB, et al. Dose escalation with three-dimensional conformal radiation therapy affects the outcome in prostate cancer. Int J Radiat Oncol Biol Phys1998; 41:491-500.

4. Zelefsky MJ, Wallner KE, Ling CC, et al. Comparison of the 5-year outcome and morbidity of three-dimensional conformal radiotherapy versus transperineal permanent iodine-125 implantation for early-stage prostatic cancer. J Clin Oncol 1999; 17:517-22.

5. Herman MG, Balter JM, Jaffray DA, et al. Clinical use of electronic portal imaging: report of AAPM Radiation Therapy Committee Task Group 58. Med Phys 2001; 28:712-37.

6. McKenzie AL, van Herk M, Mijnheer B. The width of margins in radiotherapy treatment plans. Phys Med Biol 2000; 45:3331-42.

7. Hanley J, Lumley MA, Mageras GS, et al. Measurement of patient positioning errors in three-dimensional conformal radiotherapy of the prostate. Int J Radiat Oncol Biol Phys 1997; 37:435-44.

8. Mageras GS. Introduction: management of target localization uncertainties in external-beam therapy. Semin Radiat Oncol 2005; 15:133-5.

9. International Commission on Radiation Units and Measurements (ICRU). Prescribing, Recording and reporting photon beam therapy, ICRU report 50 . Bethesda, Maryland: ICRU Publications; 1993:1-71.

10. International Commission on Radiation Units and Measurements (ICRU). Prescribing, Recording and 
reporting photon beam therapy (supplement to ICRU report 50), ICRU Report 62. Bethesda, Maryland: ICRU Publications; 1999: 1-52.

11. Murphy MJ, Balter J, Balter S, et al. The management of imaging dose during image-guided radiotherapy: report of the AAPM Task Group 75. Med Phys 2007; 34:4041-63.

12. Balter JM, Sandler HM, Lam K, et al. Measurement of prostate movement over the course of routine radiotherapy using implanted markers. Int J Radiat Oncol Biol Phys 1995; 31:113-8.

13. Acquah GF. The use of gold markers and electronic portal imaging for radiotherapy verification in prostate cancer patients: Sweden Ghana Medical Centre experience. Int J Cancer Ther Oncol 2014; 2:020112.

14. Bel A, Vos PH, Rodrigus PT, et al. High-precision prostate cancer irradiation by clinical application of an offline patient setup verification procedure, using portal imaging. Int I Radiat Oncol Biol Phys 1996; 35:321-32.

15. Dawson LA, Sharpe MB. Image-guided radiotherapy: rationale, benefits, and limitations. Lancet Oncol 2006; 7:848-58.

16. Dawson LA, Mah K, Franssen E, Morton G. Target position variability throughout prostate radiotherapy. Int J Radiat Oncol Biol Phys 1998; 42:1155-61.

17. van Lin EN, Nijenhuis E, Huizenga $H$, et al. Effectiveness of couch height-based patient set-up and an off-line correction protocol in prostate cancer radiotherapy. Int J Radiat Oncol Biol Phys 2001; 50:569-77.
18. Vigneault E, Pouliot J, Laverdière J, Roy J, Dorion M. Electronic portal imaging device detection of radio opaque markers for the evaluation of prostate position during megavoltage irradiation: a clinical study. Int J Radiat Oncol Biol Phys 1997; 37:205-12.

19. Nederveen AJ, van der Heide UA, Dehnad H, et al. Measurements and clinical consequences of prostate motion during a radiotherapy fraction. Int $J$ Radiat Oncol Biol Phys 2002; 53:206-14.

20. Poggi MM, Gant DA, Sewchand W, Warlick WB. Marker seed migration in prostate localization. Int J Radiat OncolBiol Phys 2003; 56:1248-51.

21. Hurkmans CW, Remeijer P, Lebesque JV, Mijnheer BJ. Set-up verification using portal imaging; review of current clinical practice. Radiother Oncol 2001; 58:105-20.

22. Schallenkamp JM, Herman MG, Kruse JJ, Pisansky TM. Prostate position relative to pelvic bony anatomy based on intraprostatic gold markers and electronic portal imaging. Int J Radiat Oncol Biol Phys 2005; 63:800-11.

23. Lattanzi J, McNeeley S, Donnelly S, et al. Ultrasound-based stereotactic guidance in prostate cancer--quantification of organ motion and set-up errors in external beam radiation therapy. Comput Aided Surg 2000; 5:289-95.

24. Jassal K, Munshi A, Sarkar B, et al. Validation of an integrated patient positioning system: Exactrac and iViewGT on Synergy Platform. Int J Cancer Ther Oncol 2014; 2:020212. 